\title{
Choroidal Melanoma with No Evident Metastasis on Primary Diagnosis Hitting after a Long Period - A Challenging Case
}

\author{
Pradeep Sune ${ }^{1}$, Mona Sune ${ }^{2}$, Ritica Mukherji ${ }^{3}$, Vishal Kalode ${ }^{4}$, Rajeev Pardasani ${ }^{5}$ \\ 1, 2, 3, 4, 5 Department of Ophthalmology, Datta Meghe Institute of Medical Sciences \\ (Deemed to Be University), Sawangi, Wardha, Maharashtra, India.
}

\section{INTRODUCTION}

Choroidal melanoma has been described in literature as "a complex and evolving story" which is not far from the reality. While the incidence of this deadly disease is lower in the Asian population in general, the severity of symptoms and lack of cost affordable strategies to manage a case with extensive metastasis are lacking. Therefore, it should be our primary objective as ophthalmologists to diagnose and manage the primary malignancy at the earliest and to persuade the patient for frequent follow ups.

Uveal melanoma is the most commonly found primary intraocular tumour in adults. ${ }^{1}$ Out of the three varieties, choroidal melanoma accounts for $80 \%$ of all uveal melanoma. It is more commonly found in Caucasians and in middle aged males ${ }^{2}$ with a predilection for tropical areas with high levels of solar radiation. ${ }^{3}$ The incidence of uveal melanoma in Asian and African population is $0.15-0.18$ and $0.2-0.4$ per million respectively which is lowest in the world. ${ }^{4}$ Its origin can be attributed to the melanocytes or melanin-containing cells found in uveal tissue and important predisposing factors include lighter skin tone and eye colour. Shah et al ${ }^{5}$ also stated that UV exposure due to arc welding is also a risk factor. Around one fourth of all cases maybe asymptomatic. Presentation as secondary angle closure glaucoma is rare. Othman et $\mathrm{al}^{6}$ found this rare presentation in eight cases all of whom required enucleation. In fact, glaucoma as an initial manifestation of uveal melanoma has been seen in only $3 \%$ of all cases. ${ }^{7}$

Collaborative ocular melanoma study (COMS) was one of the largest multicentric randomised trials with 1302 subjects detailing demography of patients, classification as well as a comparative evaluation of its treatment modalities. It paved the way for the current standard of care in treating ocular melanomas. This study showed the growth patterns of small sized tumour and demonstrated that there was no significant difference in long term survival rates in patients treated by enucleation and those treated by brachytherapy. ${ }^{8}$ Although considered a landmark it did not take into account the doubling time for this aggressive malignancy nor did it account for micro metastasis not easily detectable at the time of presentation of primary tumour. There was also a lack of data regarding iris and ciliary body melanomas. ${ }^{9}$ In this respect the TNM classification of uveal melanoma is more comprehensive, if not complex. The main treatment modalities are radiation therapy and surgical removal of local tumour which range from the fairly simpler enucleation and exenteration to the more challenging endo-resection, exo-resection, transscleral or transretinal resection. The treatment of metastasis is less satisfactory and includes either resection of regional metastasis or hepatic intraarterial chemotherapy or hepatic perfusions. None of which have shown to have good long-term survival.10,11,12

\author{
Corresponding Author: \\ Dr. Mona Sune, \\ Associate Professor, \\ Himalaya Vishwa Colony, \\ Plot No. M4, Nagpur Road, \\ Wardha, Maharashtra, India. \\ E-mail: drmonasune@gmail.com
}

DOI: $10.14260 / \mathrm{jemds} / 2021 / 157$

How to Cite This Article:

Sune P, Sune M, Mukherji R, et al. Choroidal melanoma with no evident metastasis on primary diagnosis hitting after a long period - a challenging case. J Evolution Med Dent Sci 2021;10(10):735-738, DOI: 10.14260/jemds/2021/157

Submission 06-10-2020,

Peer Review 01-01-2021,

Acceptance 07-01-2021,

Published 08-03-2021.

Copyright (C) 2021 Pradeep Sune et al. This is an open access article distributed under Creative Commons Attribution License [Attribution 4.0 International (CC BY 4.0)] 


\section{PRESENTATION OF CASE}

The patient is a 37 years old male, a daily wage labourer from central India. He reported to the Ophthalmology department of a tertiary care hospital with complaints of pain in left eye of acute onset associated with diminution of vision initially, which progressed to loss of projection of rays in all four quadrants over the same duration. There was accompanying continuous severe headache, throbbing in nature; photophobia and watering. He had no significant past history or family history of glaucoma.

After obtaining informed verbal consent from patient for examination and photographic documentation a detailed ocular and systemic examination was carried out.

The torchlight slit lamp and fundus examination of left eye was within normal limits with no abnormality detected. Ocular examination of right eye disclosed the following positive findings. Visual acuity decreased down to perception of light + / projection of rays inaccurate in 2 quadrants. His colour vision could not be assessed, patient had oedema over upper eyelid with conjunctival congestion, circum-corneal congestion and diffuse stromal oedema. Anterior chamber was very shallow, VH grade 1 and pupil $-3 \mathrm{~mm}$ in size, circular, and fixed. No abnormality detected in lens.
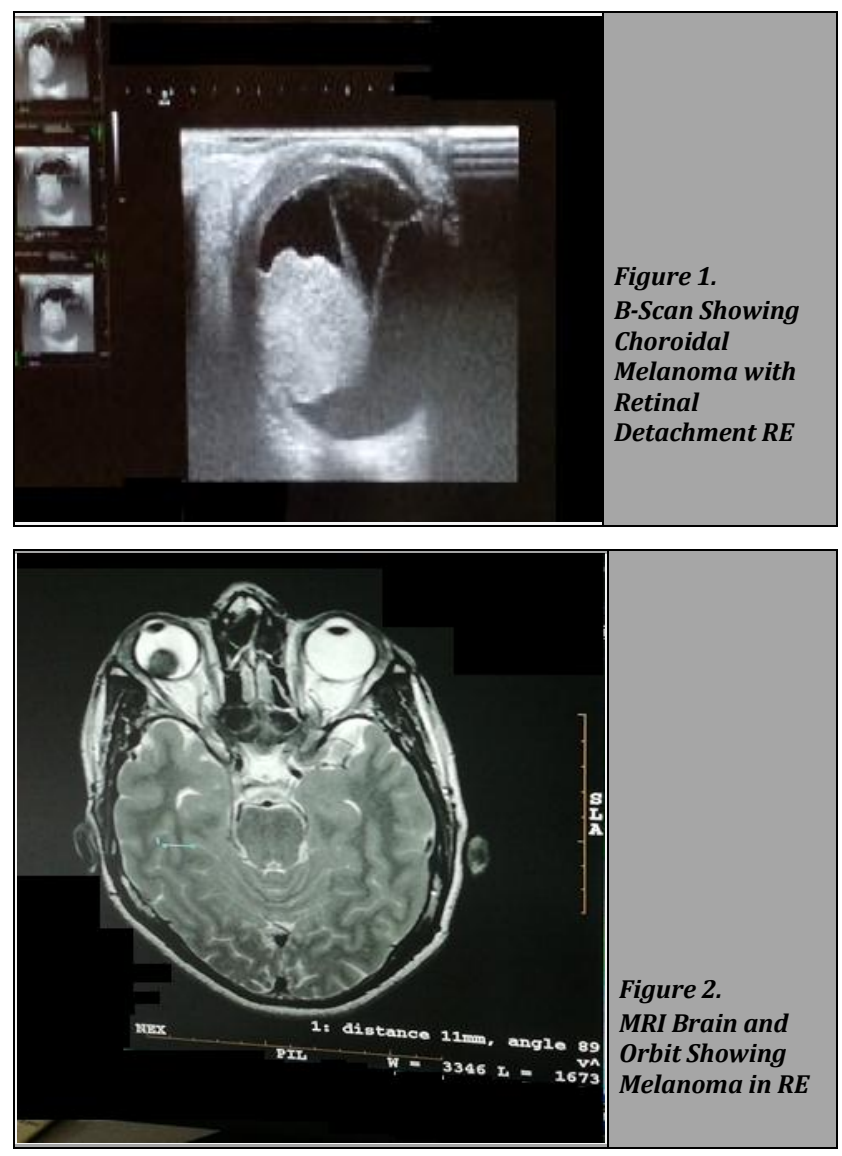

Intraocular pressure-IOP (by Schiotz tonometry) was over $40 \mathrm{mmHg}$. Extraocular movements were free and full in all directions of gaze and painless.

There was no glow on distant direct ophthalmoscopy. With no improvement in clinical picture of patient and no view of fundus, it was imperative to assess the posterior segment of the patient in order to reassess and modify the treatment.
B-scan of left eye [Fig. 1] showed evidence of a V shaped membrane attached to fovea suggestive of retinal detachment and a large hypoechoic lesion of size $15 \times 10 \mathrm{~mm}$ was seen behind the detached retina indicative of a space occupying lesion arising from choroid (possibly choroidal melanoma). An MRI was advised to confirm the soft-tissue diagnosis.

MRI brain with orbit [Fig. 2] detailed a well-defined altered signal intensity lesion noted in superior temporal quadrant of right eye-suggestive of choroidal melanoma over metastasis. The dimensions of the tumour were confirmed to be $13 \times 13.2$ $\mathrm{x} 10 \mathrm{~mm}$. There was no evidence of any extraocular extension.

Ultrasonography (USG) abdo-pelvis was done to rule out presence of liver metastasis as it is the commonest site for the same. Furthermore, a liver function test was done to rule out functional deficit corresponding to presence of metastasis. In addition, a chest $\mathrm{x}$-ray was also done to rule out metastasis to lung. All investigations were found to be within normal limits.

A positron emission tomography (PET) scan was advised but could not be done due to patient's financial constraints. LFT d included levels of gamma glutamyl transferase or alkaline phosphate were normal which have been specifically found to be raised in patients of uveal melanoma with suspected micro metastasis.

\begin{tabular}{|cccc|}
\hline T & N & M & Stage Group \\
Ti a & N0 & M0 & I \\
TIb-d & N0 & M0 & IIA \\
T2a & N0 & M0 & IIA \\
T2b & N0 & M0 & IIB \\
T3a & N0 & M0 & IIB \\
T2c-d & N0 & M0 & IIIA \\
T3b-c & N0 & M0 & IIIA \\
T4a & N0 & M0 & IIIA \\
T3d & N0 & M0 & IIIB \\
T4b-c & N0 & M0 & IIIC \\
T4d-e & N0 & M0 & IV \\
Any T & N1 & M0 & IV \\
\hline Any T & N1 & M1a-c & \\
\hline Figure 3. AJCC Classification of Posterior Uveal Melanoma & (Choroidal and Ciliary Body) Staging \\
\hline
\end{tabular}

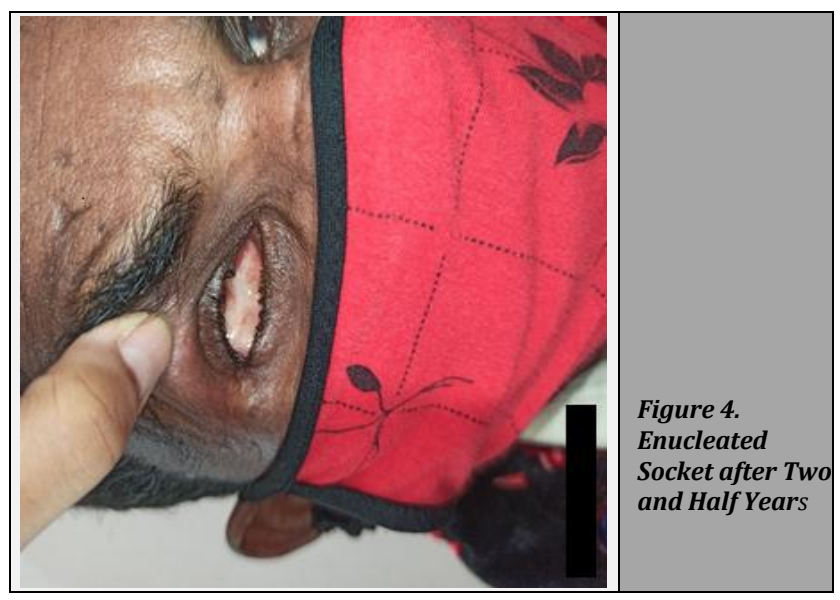

\section{DISCUSSION}

The COMS trial ${ }^{9}$ also proposed that largest basal diameter and age were important prognostication factors. MRI was useful 
for definitively proposing the tumour in this case to be of medium size based on the COMS study.

The American Joint Committee on Cancer (AJCC) classification of the tumour placed it in Stage IIIA with a TNM classification of T3B N0 M0.13 (Fig 3)

\section{DISCUSSION OF MANAGEMENT}

On admission, patient's intraocular pressure was more than 40 $\mathrm{mm}$ of $\mathrm{Hg}$ using non-contact tonometer. Patient was given intravenous mannitol $1 \mathrm{~g} / \mathrm{Kg}$ stat followed by tablet acetazolamide $250 \mathrm{mg}, 2$ tablets stat followed by 1 tablet TDS with syrup K-sol, eyedrop pilocarpine $1 \%$ QID, eyedrop combination of brimonidine $0.15 \%$ and timolol $0.5 \%$ BD. There was no symptomatic improvement after three days of therapy.

Enucleation of right eye was planned in view of painful blind eye and no evidence of extraocular extension or metastasis. Care was taken to cut the long stump of optic nerve (at least $10 \mathrm{~mm}$ globe was removed from the socket and sent for histopathology under standard preservation. Tenon's and conjunctiva were closed with running sutures.

Post-operatively patient was started on analgesics, antacids and antibiotic. There were no post-operative complications. Except for ptosis. Patient was discharged on third postoperative day and 10 days after admission with instructions to follow up after 15 days.

\section{Follow-Up and Outcomes}

The histopathology report of the patient detailed the following-

- On cut-section of specimen a jet-black tumour was seen in anterior and posterior chamber approximately $2 \times 2 \mathrm{~cm}$ in size.

- Histology was evocative of choroidal melanocarcinomaepitheloid type, extending within sclera.

- The collapsed area of the tumour shows partially detached retina.

- Optic nerve sampled in the section was free of tumour cell infiltrate.

Patient was asked for follow up every three months as there was no evident metastasis on various radiological investigations and biochemical markers in view of micrometastasis. Unfortunately, thereafter patient did not follow up in eye OPD for a long period of two and half years.

He presented to the gastroenterology OPD two and half years after primary ocular intervention with complaints of abdominal pain. A contrast enhanced computed tomography (CT) of abdomen was advised which revealed hepatomegaly with extensive metastasis. This was followed by a fine needle aspiration cytology (FNAC) of liver which showed cytomorphological features suggestive of "deposits of epithelial malignancy" [Melanoma].

In view of this the diagnosis of the patient was revised to incorporate these new findings. At present, patient is an operated case of choroidal melanoma stage IV undergoing palliative chemotherapy with dacarbazine $375 \mathrm{mg} / \mathrm{m}^{2}$ three weekly with a poor prognosis.

There is no evidence of recurrence at the primary site and / or in the fellow eye currently. The enucleated eye shows signs of healthy fibrosis. [Fig. 4]

\section{FINAL DIAGNOSIS}

Medium sized melanotic choroidal melanoma. Epitheloid variant, measuring $13.2 \times 13 \times 10 \mathrm{~mm}$ in superotemporal quadrant with secondary angle closure glaucoma, with exudative retinal detachment and no extraocular extension. TNM Grading - T3B N0 M0. Stage IIIA

\section{DISCUSSION}

This report illustrates that in a case of unilateral acute angle closure, a differential of intraocular tumour should be considered, diagnosed definitively and treated at the earliest in order to avoid life threatening and vision threatening complications. Contrary to expectation, a tumour with an incidence as low as uveal melanoma in India, chances are that classical features of the tumour may be overlooked or attributed to different diagnoses contributing to late presentations. This means that a patient may present to us when micro metastasis is already in place.

In a study detailing rates of metastasis following diagnosis and treatment of primary choroidal melanoma, Koutsandrea and associates ${ }^{14}$ found that the median interval of time between diagnosis of primary and metastasis was 29.5 months. They also found that patients with larger tumours were frequent candidates of enucleation and that larger tumours were associated with a greater risk of development of metastasis.

Posterior uveal melanomas disseminate through vortex veins into the blood flow. Most metastasis are found in liver (92\%), but the tumour can also invade the lungs and skin.

There is significant evidence that choroidal melanomas may arise from choroidal naevi, since naevi cells have been found in histopathological samples of melanomas. ${ }^{15,16}$

"To Find Small Ocular Melanoma Using Helpful Hints Daily". If three or more of these factors are present, they estimated a greater than $50 \%$ risk of malignant transformation.

- T: Thickness $>2 \mathrm{~mm}$.

- F: Fluid (subretinal).

- S: Symptoms.

- 0: Orange pigment (presence of).

- M: Margin within $3 \mathrm{~mm}$ of the optic disc.

- U: Ultrasonographic.

- H: Hollowness.

- H: Absence of surrounding halo. Nevi usually show a surrounding clear halo consisting of atrophied retina.

- D: Absence of drusen. 
Naevi account for the commonest cause of misdiagnosis of a choroidal melanoma and is referred to as a" pseudomelanoma". ${ }^{9}$

Even though the case on hand appeared to be diagnosed and managed definitively prior to systemic dissemination, it must be remembered that choroidal melanoma is an aggressive tumour and that all reasonable attempts should be made to sensitize the patient to a poor outcome despite primary surgery and emphasize the need for bi-annual liver function tests and annual radiographic examination to rule out metastasis.

Clinically, patients with a medium to large sized tumour having a histological diagnosis of "epitheloid" variant have a much larger risk of metastasis. ${ }^{1}$

In addition, a genetic diagnosis should be attempted in order to understand the risk of metastasis. Genetic profiling of the patients using in-situ hybridization technique has allowed us to identify cases with high potential for metastasis. Loss from chromosome 3 or monosomy and gain in chromosome 8 have been associated with a greater risk of metastasis so much so that loss from chromosome three has been found to reduce survival rates from $100 \%$ to $50 \% .^{17,18,19}$ Gain in chromosome 6 have better prognosis. A microRNA profiling has also found monosomy of chromosome 3 to have a higher metastatic potential. 20,21

In conclusion, the two take-aways from this case are to look out for atypical presentations of uveal melanomas and to counsel patient diligently regarding follow up in order to maximise visual prognosis and survival rates.

Financial or other competing interests: None.

Disclosure forms provided by the authors are available with the full text of this article at jemds.com.

\section{REFERENCES}

[1] Kanski J, Bowling B. Kanski's Clinical Ophthalmology: a systematic approach. $8^{\text {th }}$ edn. Saunders Ltd., 2016.

[2] Singh AD, Turell ME, Topham AK. Uveal melanoma: trends in incidence, treatment and survival. Ophthalmology 2011;118(9):1881-5.

[3] Seddon JM, Gragoudas ES, Glynn RJ, et al. Host factors, UV radiations and risk of uveal melanoma. A casecontrol study. Arch Ophthalmol 1990;108(9):1274-80.

[4] Kivelä TT. Incidence, prevalence and epidemiology of ocular melanoma. In: Murray TG, Boldt HC, eds. Ocular melanoma: advances in diagnostic and therapeutic strategies. $1^{\text {st }}$ edn. London: Future Medicine Ltd., 2014: p. 20-38.

[5] Shah CP, Weis E, Lajous M, et al. Intermittent and chronic ultraviolet light exposure and uveal melanoma: a metaanalysis. Ophthalmology 2005;112(2):1599-607.

[6] Othman IS, Assem M, Zaki IMA. Secondary glaucoma as initial manifestation of uveal melanoma. Saudi J Ophthalmol 2013;27(3):203-8.
[7] Shields CL, Materin MA, Shields JA, et al. Factors associated with elevated intraocular pressure in eyes with iris melanoma. Br J Ophthalmol 2001;85(6):666-9.

[8] The collaborative ocular melanoma study (COMS) randomized trial of pre-enucleation radiation of large choroidal melanoma I: characteristics of patients enrolled and not enrolled. COMS report no. 9. Am J Opthalmol 1998;125(6):767-78.

[9] Pal BP, Garge S, Khetan V. Choroidal melanoma: a short review with an Indian perspective. Oman J Ophthalmol 2017;10(3):135-44.

[10] Gupta S, Bedikian AY, Ahrar J, et al. Hepatic artery chemoembolization in patients with ocular melanoma metastatic to the liver: response, survival and prognostic factors. Am J Clin Oncol 2010;33(5):474-80.

[11] Patel K, Sullivan K, Berd D, et al. Chemoembolization of the hepatic artery with BCNU for metastatic uveal melanoma: results of a phase II study. Melanoma Res 2005;15(4):297-304.

[12] Schuster R, Lindner M, Wacker F, et al. Transarterial chemoembolization of liver metastases from uveal melanoma after failure of systemic therapy: toxicity and outcome. Melanoma Res 2010;20(3):191-6.

[13] Kivelä T, Simpson RE, Grossniklaus HE, et al. Uveal melanoma. In: AJCC Cancer Staging Manual. $8^{\text {th }}$ edn. New York, NY: Springer 2016: p. 805-17.

[14] Koutsandrea C, Moschos MM, Dimissianos M, et al. Metastasis rates and sites after treatment for choroidal melanoma by proton beam irradiation or by enucleation. Clin Ophthalmol 2008;2(4):989-95.

[15] Shields CL, Dalvin LA, Ancona-Lezama D, et al. Choroidal nevus imaging features in 3,806 cases and risk factors for transformation into melanoma in 2,355 cases. Retina 2019;39(10):1840-51.

[16] Singh AD, Kalyani P, Topham A. Estimating the risk of malignant transformation of a choroidal nevus. Ophthalmology 2005;112(10):1784-9.

[17] Field MG, Harbour JW. Recent developments in prognostic and predictive testing in uveal melanoma. Curr Opin Ophthalmol 2014;25(3):234-9.

[18] van Essen TH, van Pelt SI, Versluis M, et al. Prognostic parameters in uveal melanoma and their association with BAP1 expression. Br J Ophthalmol 2014;98(12):1738-43.

[19] Coupland SE, Lake SL, Zeschnigk M, et al. Molecular pathology of uveal melanoma. Eye (Lond) 2013;27(2):230-42.

[20] Radhakrishnan A, Badhrinarayanan N, Biswas J, et al. Analysis of chromosomal aberration (1, 3 and 8) and association of microRNAs in uveal melanoma. Mol Vis 2009;15:2146-54.

[21] Venkatesan N, Kanwar J, Deepa PR, et al. Clinicopathological association of delineated miRNAs in uveal melanoma with monosomy 3/disomy 3 chromosomal aberrations. PLoS One 2016;11(1):e0146128. 